\title{
Improving Throughput and Microstructure Uniformity in Direct Laser Interference Patterning Utilizing Top-Hat Shaped Beams
}

Mikhael El-Khoury ( $\square$ mikhael.el-khoury@tu-dresden.de )

TU Dresden

\section{Bogdan Voisiat}

TU Dresden

\section{Tim Kunze}

Fraunhofer Institute for Material and Beam Technology

Andrés Fabián Lasagni

TU Dresden

\section{Research Article}

Keywords: Direct Laser Interference Patterning (DLIP), fundamental beam-mode shaper (FBS), microstructures, Gaussian intensity

Posted Date: February 12th, 2021

DOI: https://doi.org/10.21203/rs.3.rs-183663/v1

License: (9) This work is licensed under a Creative Commons Attribution 4.0 International License. Read Full License 


\title{
Improving throughput and microstructure uniformity in Direct Laser Interference Patterning utilizing top-hat shaped beams
}

\author{
Mikhael El-Khoury ${ }^{1}{ }^{*}$, Bogdan Voisiat ${ }^{1}$, Tim Kunze $^{2}$, Andrés Fabián Lasagni ${ }^{1,2}$ \\ 1 Institut für Fertigungstechnik, Technische Universität Dresden, George-Baehr-Str. 3c, 01069 \\ Dresden, Germany \\ 2 Fraunhofer-Institut für Werkstoff- und Strahltechnik IWS, Winterbergstr. 28, 01277 Dresden, \\ Germany \\ * mikhael.el-khoury@tu-dresden.de
}

\begin{abstract}
Uniform periodic microstructures formation over large areas is generally challenging in Direct Laser Interference Patterning (DLIP) due to the Gaussian laser beam intensity distribution inherent to most commercial laser sources. In this work, a diffractive fundamental beam-mode shaper (FBS) element is implemented in a four-beam DLIP optical setup to generate a square-shaped top-hat intensity distribution in the interference volume. The interference patterns produced by a standard configuration and the developed setup are measured and compared. In particular, the impact of both laser intensity distributions on process throughput as well as fill-factor is investigated by measuring the resulting microstructure height with height error over the structured surface. It is demonstrated that by utilizing top-hat-shaped interference patterns, it is possible to produce on average $44.8 \%$ deeper structures with up to $60 \%$ higher homogeneity at the same throughput. Moreover, the presented approach allows the production of microstructures with comparable height and homogeneity compared to the Gaussian intensity distribution with increased throughput of $53 \%$.
\end{abstract}

\section{Introduction}

In recent years, laser surface texturing (LST) has proven to be a suitable tool for producing various surfaces with controllable topography, leading to improved surface properties, such as wettability ${ }^{1,2}$ and self-cleaning ${ }^{3}$, tribology ${ }^{4}$ and antifouling properties ${ }^{5}$. Nowadays, out of the available LST methods, Direct Laser Interference Patterning (DLIP) has arisen as an innovative and effective tool for high throughput surface micro-structuring ${ }^{6-8}$. This technique enables a direct fabrication of flexible and perfect periodic surface patterns with a well-defined long-range order based on the interference principle $^{9-12}$. In addition, it has been shown that the number of interfering laser beams, their geometrical arrangement, individual angles, phase and polarization influence the shape of the interference pattern as well as its typical repetitive distance (spatial period) ${ }^{13-14}$. Due to the flexibility to achieve highly complex patterns in a one-step process, DLIP is especially interesting for industrial applications. Moreover, no chemicals, post-treatments or vacuum conditions are required, making it an eco-friendly, fast and cost-optimized process ${ }^{12}$.

During DLIP processing, the material interacts with the laser radiation predominantly at the positions corresponding to the interference maxima, inducing various metallurgical processes such as melting, ablation and recrystallization ${ }^{15,16}$. During nanosecond-pulsed laser processing of metals, the structuring mechanism is mainly based on recoil vapour pressure and Marangoni convection, that have an effect on the overall picture of melt flow ${ }^{17,18}$. Since the local intensity at the interference maxima positions is directly related to the intensity distribution in the laser beam profile, the use of a round Gaussian beam leads to inhomogeneous surface textures due to the non-uniform intensity distribution of the input laser beam ${ }^{19,20}$. However, most commercial lasers provide beams with Gaussian (TEM00) intensity distribution. This intensity profile preserves its distribution during propagation, and it can be focused to a diffraction-limited spot. Furthermore, the energy distribution of the Gaussian beam gradually decreases from the centre to the boundary of the laser spot. Consequently, the spot area limited by a beam diameter (at $1 / \mathrm{e}^{2}$ level) includes only $86.5 \%$ of the laser beam energy, and the intensity at the boundary is only $13.5 \%$ of the peak intensity ${ }^{20}$. Moreover, previous investigations showed that only $36.8 \%$ of the pulse energy is used efficiently at the focal position for a Gaussian beam ${ }^{21,22}$. In particular, the energy per unit of area (fluence) at the tails can be much lower than the ablation threshold leading to undesired heating effects of the surface surrounding 
the laser spot without any ablation (see Fig. 1a). In addition, the excess energy located at the centre of the beam can lead to uncontrolled melting (over melting), which in turn affects the microstructure quality. Consequently, nearly two-thirds of the used laser power of a Gaussian beam is potentially wasted during laser micro-structuring process.

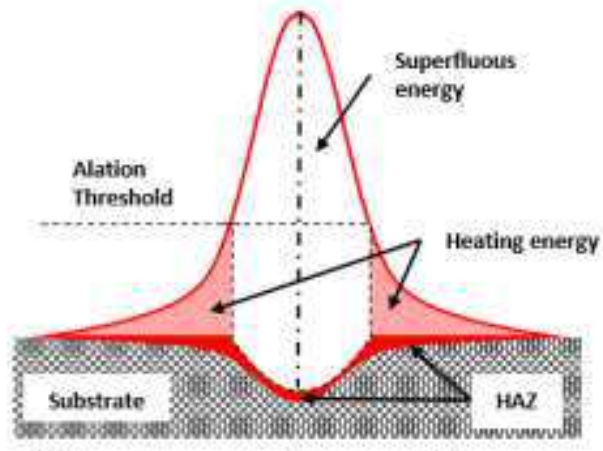

(a) Laser gaussian beam profile on substrate surface

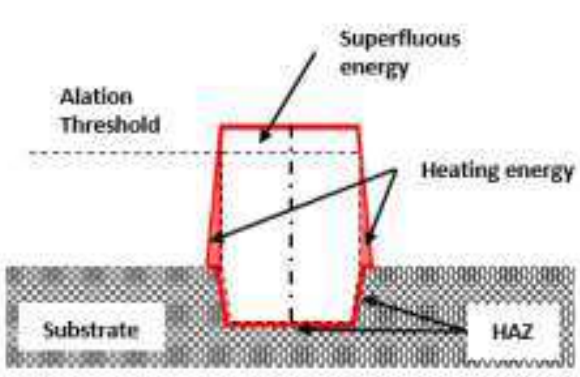

(b) Laser top-hat beam profile on substrate surface
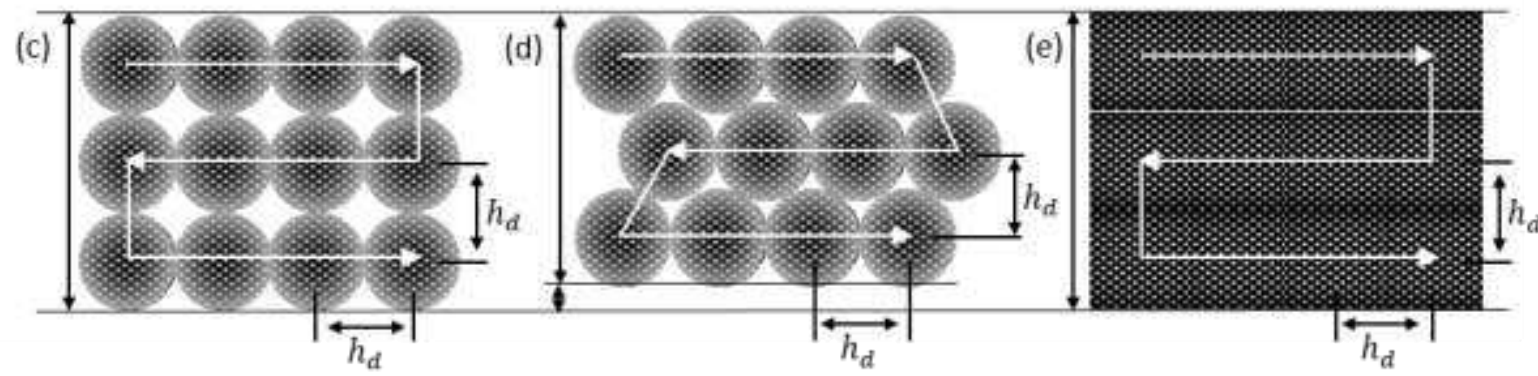

Figure 1. Laser energy contribution to material ablation and formation of Heat affected Zone (HAZ) when (a) Gaussian and (b) top-hat beams are utilized ${ }^{23}$. Illustration of structured area coverage during DLIP with (c) square and (d) hexagonally distributed Gaussian laser spots as well as (e) top-hat shaped laser beam. $h_{d}$ denotes a hatch distance between two consecutive pulses.

Another disadvantage of the Gaussian beam profile is the footprint of the ablated zone, which has a round shape and causes both an inhomogeneous profile along the processing line and a low fillfactor $^{23}$ (see Fig.1c,d). This is usually circumvented by an increase of the pulse-to-pulse overlap that leads to an improved pattern homogeneity but at the same time to lower throughputs and an increased Heat Affected Zone (HAZ) ${ }^{20,23}$. Therefore, to decrease the HAZ as well as to improve the process throughput, it is necessary to reshape the Gaussian laser beam into a uniform square-shaped intensity distribution with a top-hat geometry (Fig. 1b, e).

This study describes the development of an optical configuration to generate interference patterns, using square-shaped beams with a top-hat laser intensity distribution. This is reached by implementing a fundamental beam-mode shaper (FBS) ${ }^{24,25}$ applied to a four-beam DLIP setup ${ }^{26}$. The interference patterns produced by the symmetrical four-beam DLIP setup with Gaussian and top-hat intensity distributions are compared. The impact of the laser fluence and hatch distance on process throughput as well as on the filling factor of the structured area, the resulting microstructure height as well as the height error across the structured surface is analysed and compared.

\section{Materials and Methods}

Sample preparation. The laser texturing experiments were performed on flat samples of stainless steel (AISI 304) having a thickness of $0.7 \mathrm{~mm}$ and average surface roughness (Ra) of $52 \mathrm{~nm}$. The substrates were used as received.

Principle of a fundamental beam-mode shaper. The fundamental beam-mode shaper is a diffractive optical element, which generates a square top-hat intensity distribution at the focal plane of the focusing lens by redistributing irradiance and phase of the wave front 20,25 . The depth of focus is maintained and comparable to the Rayleigh length of the focused Gaussian beam without the FBS element ${ }^{20,25}$. The efficiency of the FBS element is $95 \%$ in an area limited by $1 / \mathrm{e}^{2}$ level, where only $5 \%$ of the laser energy is wasted in the tail region. The areal uniformity of the top-hat profile is $\pm 2.5 \%$. The 
FBS approach requires specific input laser beam parameters. The input laser beam must be high beam quality (TEM00, $\mathrm{M}^{2}<1.4$ ) with a particular beam diameter of $4 \mathrm{~mm}$ with $\pm 5 \%$ tolerance 20,25 .

Nanosecond four-beam-DLIP setup and process strategy. For the fabrication of the periodic structures, a four-beam interference setup was utilized, obtaining interference patterns with a dot-like morphology. A schematic representation of the experimental DLIP setup is shown in Fig. 2a, which includes a nanosecond (ns)-pulsed laser system (Laser Tech 1053 Advanced, Laser Export) operating at a wavelength of $1053 \mathrm{~nm}$ with a maximum average output power of $4 \mathrm{~W}$ (@4 kHz repetition rate). The FBS beam shaper (TOPAG Lasertechnik $\mathrm{GmbH}$ ) was integrated prior to the four-beam DLIP optical configuration ${ }^{27}$.

(a)

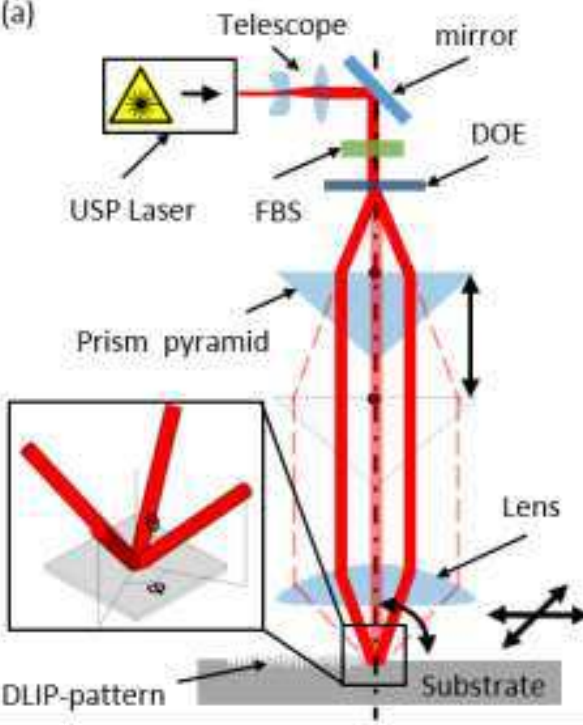

(b)

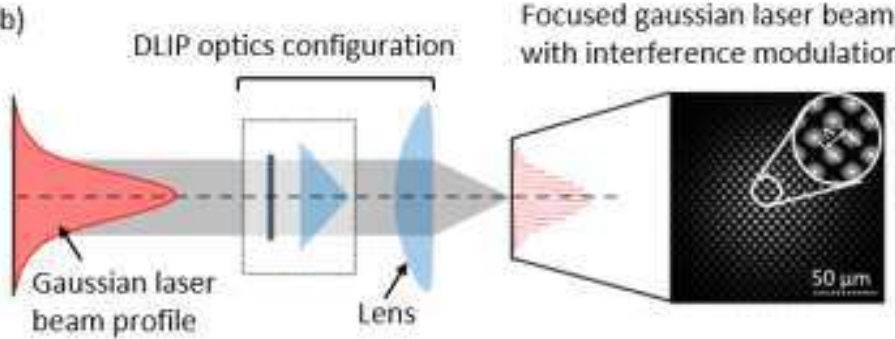

(c)

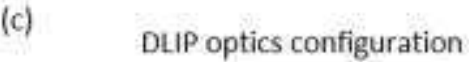

Focused top-hat laser beam with interference modulation

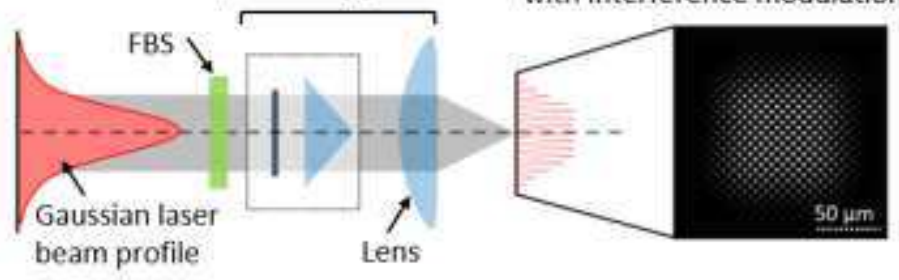

Figure 2. (a) Schematic representation of the experimental setup for structuring dot-like pattern using DLIP optical configuration combined with an FBS element; the inset at the sample surface shows the sketch of four symmetrically distributed interfering sub-beams. The incidence angle $\theta$ is the same for all four beams. The azimuthal angle $\phi$ between each consecutive beam is $90^{\circ}$ and the spatial period can be calculated by $A_{4}=\lambda /(\sqrt{2} \cdot \sin \theta)$, where $\lambda$ is a laser wavelength. Representation and measurement of the interference pattern distribution (with a beam profiling camera) for the setup without (b) and with (c) the FBS element.

The DLIP setup consists of a diffractive optical element (DOE) that splits the main laser beam into four sub-beams, a beam-parallelizing pyramid (with an apex angle of $138.4^{\circ}$ ) and a condenser lens (with an effective focal length of $100 \mathrm{~mm}$ ) for beam superposition. The four sub-beams are symmetrically distributed around the optical axis, as depicted in Fig. 2a. A confocal telescope with a 4x magnification was used to provide an input beam at the FBS element with diameter of $4.0 \mathrm{~mm}$. The resulting interference intensity pattern consists of periodically distributed peaks (DLIP pattern) with square symmetry, as shown in Fig. 2b,c.

The laser fluence (or energy density), defined as the laser energy deposited per unit of area, is one of the most important parameters that control the morphology and quality of the periodic patterns ${ }^{18,28}$. In this study, the laser fluence $\mathrm{F}_{p}$ was varied from $2.1 \mathrm{~J} / \mathrm{cm}^{2}$ up to $2.97 \mathrm{~J} / \mathrm{cm}^{2}$. The beam radius, determined using the D-squared method, was $85.7 \mu \mathrm{m}^{29}$. To structure larger areas, the sample was moved using XY-stages (PRO Series, Aerotech Ltd.) with linear speeds ranging from $0.22 \mathrm{~cm} / \mathrm{s}$ to $1.60 \mathrm{~cm} / \mathrm{s}$. The arrangement of the pulse positioning during the structuring process is illustrated schematically in Fig. $1 \mathrm{c}$-e. In this way, the laser pulses were shifted and overlapped in the $\mathrm{x}$ and $\mathrm{y}$ directions in such a way that the hatch distance $\left(h_{d}\right)$ was always kept as a multiple of the spatial period $\left(\Lambda_{4}=6.5 \mu \mathrm{m}\right)$ to guarantee a well-defined periodic structure (ranging from $71.5 \mu \mathrm{m}$ up to $104.0 \mu \mathrm{m}$ ). The maximum throughput (Thr) is proportional to the hatch distance and it can be calculated by Eq. 1:

$$
\operatorname{Thr}\left[\mathrm{cm}^{2} / \mathrm{min}\right]=h_{d^{2}} f
$$

where $f$ is the used repetition rate. 
Surface characterization. To describe the uniformity of the fabricated structures, two topographical parameters were used, namely the structure height error and the filling factor (short: fill-factor). The last describes the ratio between surface completely covered with microstructures and actual structured surface. For this purpose, the surface topography of the structured samples was characterized using a confocal microscope (Sensofar, S Neox non-contact 3D Surface Profiler) employing a 50x magnification objective, with lateral and vertical resolutions of $340 \mathrm{~nm}$ and $4 \mathrm{~nm}$, respectively. Using this objective, a total area of $351 \mu \mathrm{m} \times 264 \mu \mathrm{m}$ were recorded in each measurement. Afterward, using the software MountainsMap $\AA 7.4$, the surface profiles of the measured topographies are extracted applying morphological filters (ISO16610-14), and the topographical 3D roughness parameters are calculated (ISO 25178-2). A morphological filter is based on two morphological operations, dilation and erosion, that work using a structuring element (SE) of a given size ${ }^{29}$. In this case, the SE was set to the size of the structure period $\Lambda$. By applying the dilation and erosion morphological filtering, the upper and lower structure envelopes were calculated, respectively. The upper and lower envelopes represent the distribution of heights and depths of structure hills and valleys, respectively. Accordingly, the fabricated structure height distribution $(\mathrm{H})$ is achieved by subtracting the lower from the upper envelope. The average structure height and its error $\left(h_{\text {err }}=\left(S_{q} / S_{\text {mean }}\right) \cdot 100 \%\right)$ can be calculated by finding an average $\left(S_{\text {mean }}\right)$ and root mean square $\left(S_{q}\right)$ of $\mathrm{H}$, respectively ${ }^{29}$.

In addition, topographical measurements have been carried out using a scanning electron microscope at an operating voltage of $15 \mathrm{kV}$ (JEOL, JSM 6610LV). The captured images were analysed using ImageJ software (Java-based software developed by National Institutes of Health). The fill factor was determined by the following steps: (i) binarization (8 bit), (ii) application of a threshold (>55.20\%) and (iii) pixel interpretation (number of white and black pixels) employing the Otsu method 30 .

\section{Results and discussion}

The steel surface was firstly irradiated with laser spots forming compact and regular square arrays with a hatch distance $h_{d}=130 \mu \mathrm{m}$ in order to achieve separated areas to be analysed individually. The effect of beam reshaping from Gaussian to a top-hat beam was analysed by means of the structure height, structure error and ablated spot size as mentioned in the previous section.

Figure $3 \mathrm{a}$ and $3 \mathrm{~b}$ show regular ordered hole-like patterns with a spatial period of $\Lambda_{4}=6.5 \mu \mathrm{m}$ on the steel surface, irradiated with one pulse per spot at a laser fluence of $3 \mathrm{~J} / \mathrm{cm}^{2}$ using both Gaussian and top-hat beam profiles, respectively. In Figure 3, also the surface profiles of the produced topographies are shown, which follow the laser beam intensity distribution showed in Fig. $2 b$ and $2 c$, respectively (beam profiling camera measurements).

The obtained results for both configurations are clearly different. In case of the DLIP setup without the FBS element, due to the Gaussian distribution of the laser beam intensity, the ablated spots are rounded and the structure depth decreases gradually, going from the center to the edges of the spot (Fig. 3a). Differently, the patterns produced with the top-hat intensity profile exhibit approximately the same structure depth all over the square shaped spot (Fig. 3b). Besides material ablation during nanosecond DLIP processing, redeposition of the molten material driven by Marangoni convection and recoil vapor pressure ${ }^{17,31}$ takes place, which results in surface structures surrounded by recast material and hence leading to higher aspect ratios. It has to be also mentioned that during four-beam DLIP, all round shaped holes are formed simultaneously at the interference maxima positions. Therefore, the flow of molten material from interference maxima to minima positions which results in a merge of recast material at the interference minima positions surrounding the holes ${ }^{31,32}$. In case of the Gaussian beam, the amount of recast material decreases close to the spot edge due to the lower laser intensities. On the contrary, it forms high peaks due to superflous energy in the middle of the Gaussian beam (see surface profile in Fig. 3a). In consequence, the inhomogeneous laser intensity lead to a reduced uniformity of the final structure. On the other hand, in of the top-hat beam distribution, the recast material is formed around the holes with roughly the same height (see profile in Fig. 3b) due to the even intensity distribution in the beam profile. 


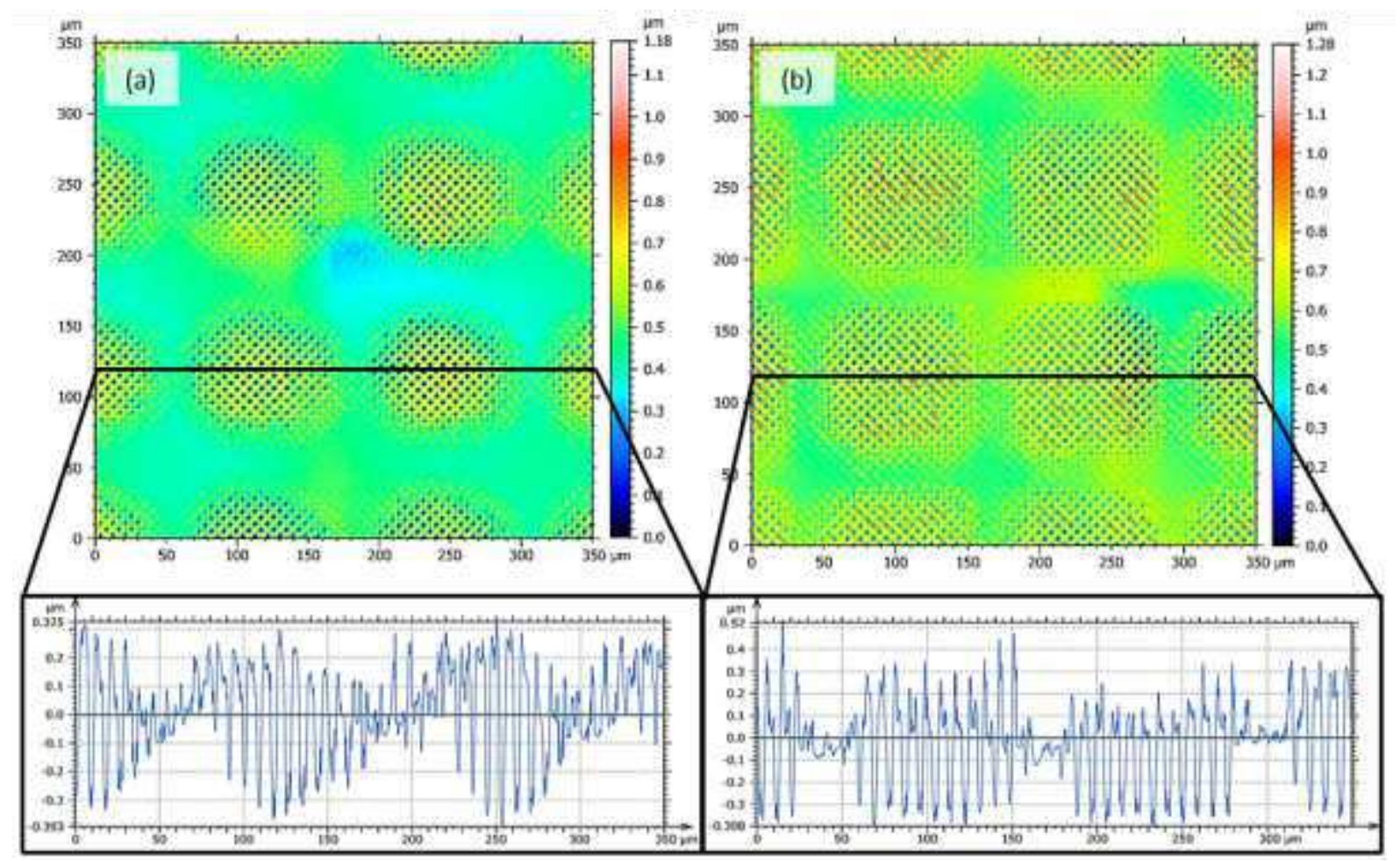

Figure 3. Confocal microscope images of surface topographies produced in stainless steel and corresponding surface profiles using the (a) Gaussian and (b) top-hat beam profiles. Only one laser pulse was used for each spot. The laser fluence was $2.97 \mathrm{~J} / \mathrm{cm}^{2}$ and the hatch distance was $130 \mu \mathrm{m}$.

Figure 4 shows the effect of the laser fluence variation on the characteristics of topographies structured with Gaussian and top-hat laser beams. In the case of structures fabricated using the FBS element, it was observed that the produced patterns were significantly higher for the used laser fluences compared to the standard configuration. The achieved structure mean height was ranging from $0.135 \mu \mathrm{m}$ to $0.395 \mu \mathrm{m}$ and from $0.480 \mu \mathrm{m}$ to $0,630 \mu \mathrm{m}$ in the case of Gaussian and squareshaped top-hat beam profiles, respectively, when laser fluence was increased from $2.1 \mathrm{~J} / \mathrm{cm}^{2}$ to $2.97 \mathrm{~J} / \mathrm{cm}^{2}$ (see Fig. 4a). This means that the fabricated structures using the FBS element are in average $44.8 \%$ higher.

Moreover, the reshaping of the beam allowed producing more uniform patterns. The structure uniformity can be described in terms of average structure height error, which is plotted in Fig. 4b with respect to the applied laser fluence. It can be seen, that in the case of square-shaped top-hat profile, the height error is always significantly smaller than in the case of the Gaussian beam profile, denoting a more uniform fabricated pattern. On average, the structure height error was $48.7 \%$ smaller for the flat-top beam and is decreasing from $41 \%$ down to $15 \%$ when laser fluence was increased from $2.1 \mathrm{~J} / \mathrm{cm}^{2}$ to $2.97 \mathrm{~J} / \mathrm{cm}^{2}$. Moreover, at high laser fluence the stuctured surface homogeneity is improved by $60 \%$ when square-shaped top-hat profile was used for patterning.

The other significant difference between the two optical configurations can be noticed in the spot size dependence on the applied laser fluence plotted in Fig. 4c. For both optical configurations, the spot size depends almost linearly on the laser fluence. However, in the case of the Gaussian beam configuration, this increase is more pronounced (see slope of the fit) showing a more sensitive dependence of the spot size with the laser fluence, which is governed by the exponentially decreasing peak fluence from the centre of the spot ${ }^{29}$. The reshaped beam's intensity profile is constant across the spot area, with a sharp peak fluence drop around the edges. This drop also contributes to the variation of the spot size with the laser fluence. However, due to the sharpness of the intensity drop, this variation is much smaller. 

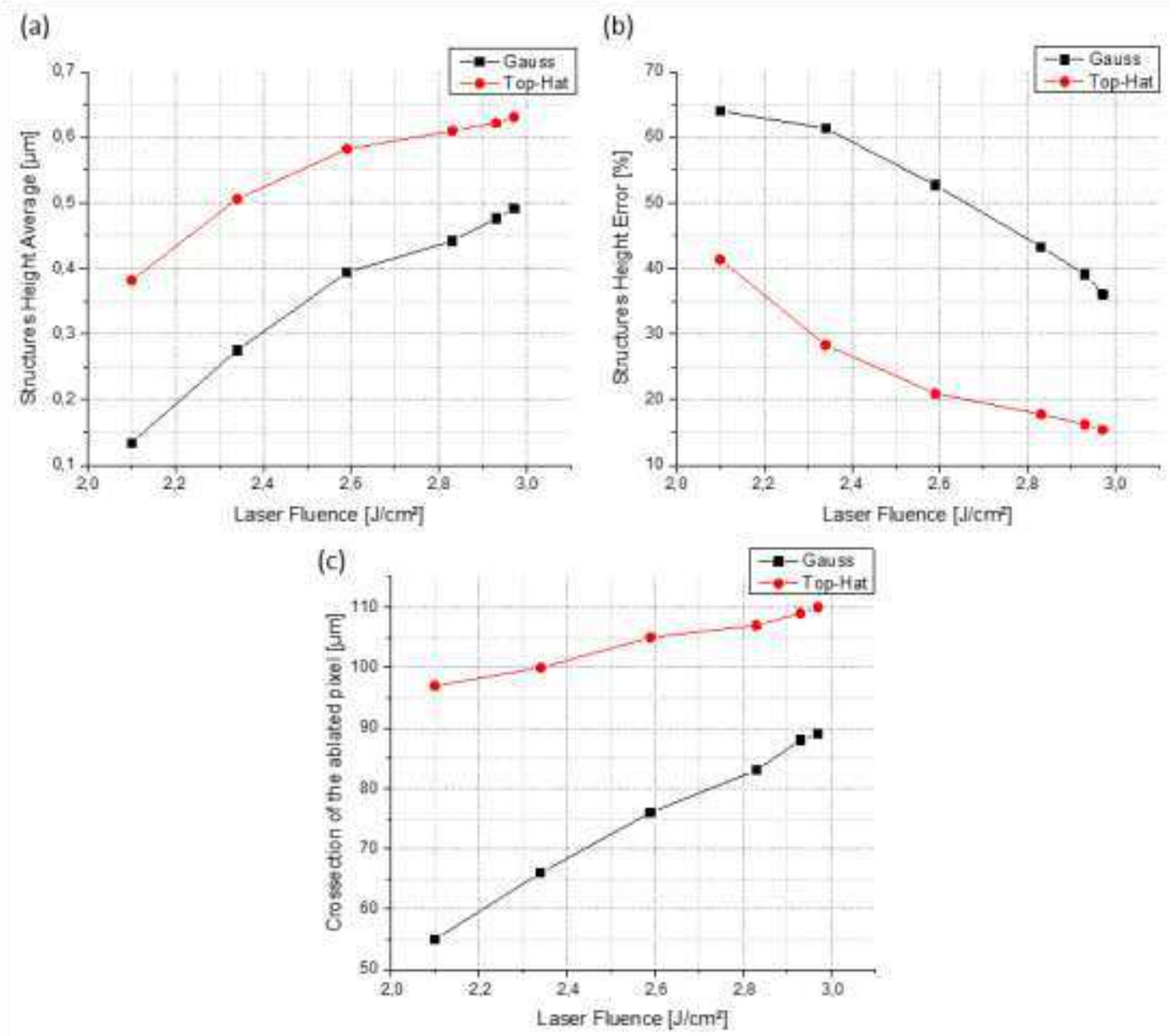

Figure 4. Graphs with topography characteristics of patterns structured with Gaussian beam and Tophat beam: (a) average structures height, (b) structures height error, (c) size of the ablated spot at different laser fluence values. The lines are used for eye guiding.

In order to investigate the beam shaping effect on large area structuring in terms of surface quality and process throughput, the hatch distance between the pulses was also varied in the range of $71.5 \mu \mathrm{m}$ and $104 \mu \mathrm{m}$. The laser fluence was also varied from $2.1 \mathrm{~J} / \mathrm{cm}^{2}$ to $2.97 \mathrm{~J} / \mathrm{cm}^{2}$. Finally, matrices containing structured areas $(3 \mathrm{~mm} \times 3 \mathrm{~mm}$ in size) were fabricated for three different fabrication conditions: i) Gaussian beam interference with a square distribution of ablated spots; ii) Gaussian beam interference with the triangular distribution of ablated spots; and iii) square-shaped top-hat beam interference pattern with a square distribution of the ablated spots. Exemplary SEM micrographs of the fabricated patterns for each fabrication strategy are shown in Fig. 5.

The patterns shown in the first row in Fig. 5 are formed with a lower laser fluence of $2.59 \mathrm{~J} / \mathrm{cm}^{2}$ and a relatively large hatch distance of $91.0 \mu \mathrm{m}$. Differently, the patterns in the second row in Fig. 5 are formed with a maximal laser fluence of $2.97 \mathrm{~J} / \mathrm{cm}^{2}$ and smaller hatch distance of $78.0 \mu \mathrm{m}$. It can be seen that the patterns in the first row contain non-structured areas left after the DLIP process, especially when Gaussian beam is used (Fig. $5 \mathrm{a}, 5 \mathrm{~b}$ ). The smallest amount of such untreated areas is visible for the case corresponding to the shaped laser beam (Fig. $5 \mathrm{c}$ ), demonstrating the benefit of a square-shaped spot for achieving better fill factors. By increasing the laser fluence and decreasing the hatch distance (Fig. $5 \mathrm{~d}$-f), the non-structured areas also vanish in the area with triangular spot distribution patterned with the Gaussian beam (Fig. 5e). However, in the case of square spot distribution (Fig. 5d), the non-structured areas are still visible, showing the ineffectiveness of this approach in filling the area with the pattern (Fig. 5a, 5d). On the other hand, in the case of square spot distribution patterned with the shaped laser beam with increased laser fluence and decreased the hatch distance (Fig. 5f), the surface is comepletely covered with patterns and shows deeper structures. 
Gaussian, Square Orientation
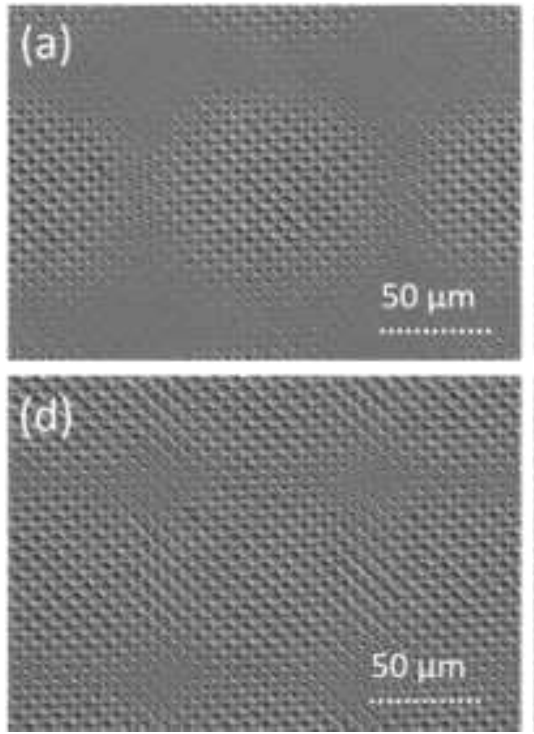

Gauss, Triangular Orientation
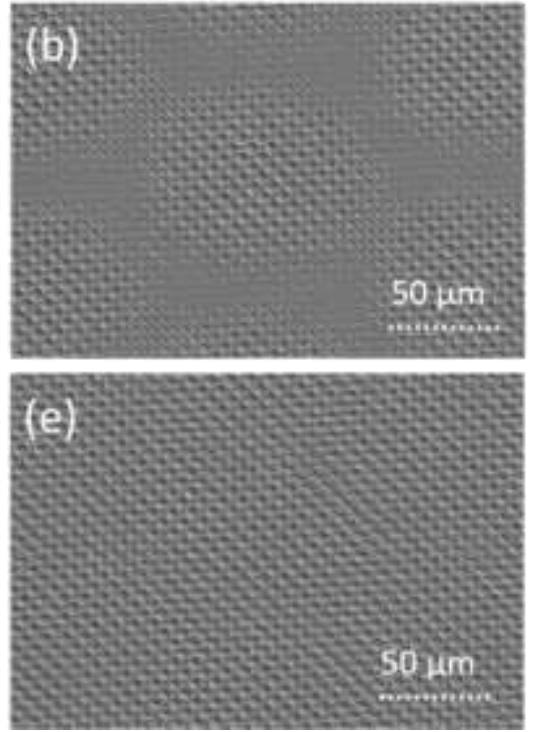

Top-Hat
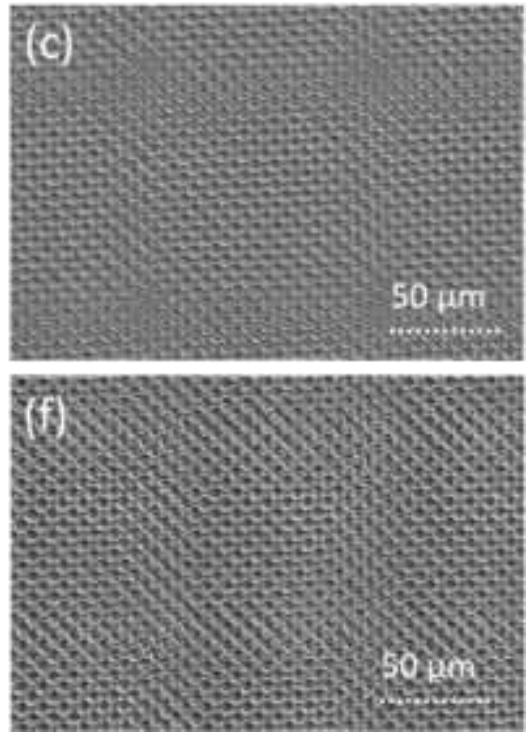

Figure 5. Scanning electron microscope (SEM) images of textured surfaces using the nanosecond four-beam DLIP configuration, produced on 1.4301 steel. The spatial period $\Lambda$ was $6.50 \mu \mathrm{m}$ and the following process parameters were used: (a-c) $F=2.59 \mathrm{~J} / \mathrm{cm}^{2}, h_{d}=91.0 \mu \mathrm{m}$; (d-f) $F=2.97 \mathrm{~J} / \mathrm{cm}^{2}$, $h_{d}=78.0 \mu \mathrm{m}$. The patterns were produced using $(\mathrm{a}, \mathrm{d}, \mathrm{c}, \mathrm{f})$ the square and $(\mathrm{b}, \mathrm{e})$ triangular distribution of the laser spots with (a, b, d, e) Gaussian and (c, f) top-hat configurations.

Each patterned area in all three matrices was analysed with a confocal microscope and three topographical parameters were measured, namely mean structured height, its error and the fill-factor. Figure 6 depicts the summary of the measured parameters in the form of 2D contour plots, where the parameters' dependence with the applied laser fluence and hatch distance (note that the hatch distance is relevant for maximizing the fabrication throughput, as indicated by Eq. 1).

The basic trends of the measured parameters are similar for all three patterning strategies. The mean structure height and fill factor increases with increasing laser fluence and decreasing hatch distance. However, the height error behaves with the opposite behaviour, showing the smallest values at the highest laser fluence and smallest hatch distance.

The main difference between the three fabrication strategies is in the slopes and absolute values of the parameters' trend. The largest difference can be seen between the Gaussian and top-hat strategies, in particular for the fill-factor. The top-hat pattern's fill-factor grows and reaches $100 \%$ of coverage area much faster than in both other cases using the Gaussian configuration (see Fig. 6i). This tendency demonstrates again the advantage of suing square-shaped spots compared to roundshaped Gaussian beams.

The higher flat-tops' beam filling factor also contributes to the overall higher average depth of the structures formed with the shaped laser beam (see Fig. 6c), which means that the fabrication of a pattern with a specific depth can be finally produced faster with a flat-top beam. In addition, the height error of the structures fabricated with the flat-top beam setup also differs strongly from the other two structuring strategies (Fig. 6d, 6e). In this case, the height error decreases faster with increasing laser fluence and decreasing hatch distance (see Fig. 6f). Therefore, a larger process window allowing high homogeneity is possible.

Similar advantages over the square-oriented Gaussian spots can be noted for the structures fabricated with triangular oriented Gaussian spots. However, the difference is much smaller in this case if we talk about structures height (Fig. 6a, 6b) as well as structures error factor (Fig. 6d, 6e) and mainly noticeable in the fill factors (Fig. $6 \mathrm{~g}, 6 \mathrm{~h}$ ). Thus, the usage of triangular distribution of the Gaussian spots for patterning the large areas is another way of getting more homogeneous structures if the beam shaping option is not possible. 

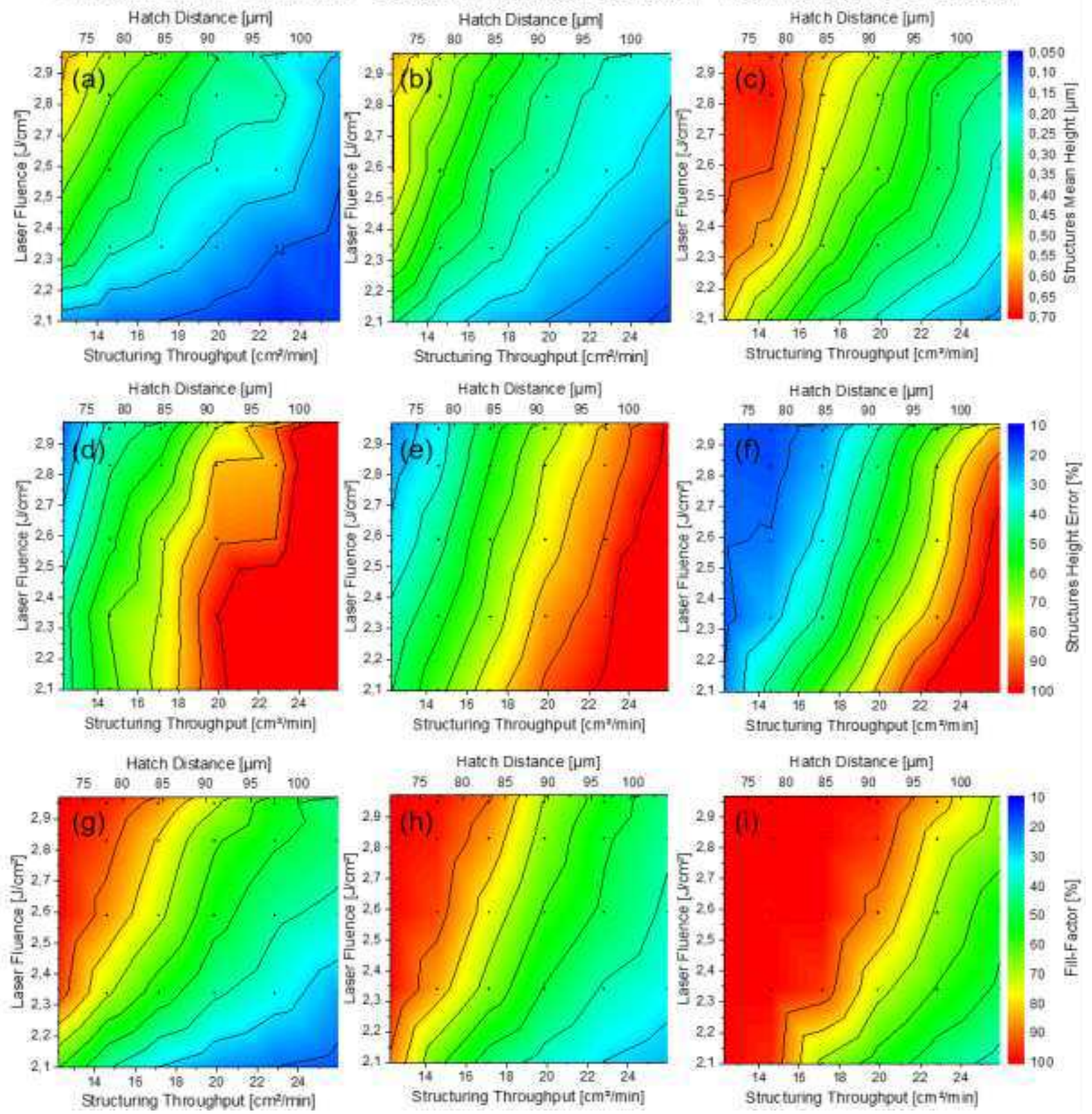

Figure 6. Contour plots of topography characteristics (structures mean height, structures height error and fill-factor) of the patterned surface with Gaussian and top-hat beam profiles as a function of laser fluence and hatch distance.

Finally, to emphasize the advantage of using a reshaped top-hat laser beam in DLIP, the process throughput for fabricating periodic patterns with certain quality was calculated. As an example, we considered a target to produce periodic structures with a fill factor over $80 \%$, and the laser fluence was set to $2.97 \mathrm{~J} / \mathrm{cm}^{2}$. The results are presented in Fig. 7 .

As it can be observed, depending on the needed structure height, different throughputs are possible. For example, for a structure height of $0.5 \mu \mathrm{m}$ a speed of $\sim 20.0 \mathrm{~cm}^{2} / \mathrm{min}$ can be reached with the tophat beam profile, while for the Gaussian beam with either square or triangular arrangements, the throughput is $\sim 14.5 \mathrm{~cm}^{2} / \mathrm{min}$. Hence it is possible to pattern the surface $\sim 43 \%$ faster if the top-hat beam profile is used. Moreover, if higher structures are needed, the top-hat configuration allows avoiding an excess of deposited intensity on the processed surface, which is important for decreasing uncontrolled melt of the material and thus lower qualities. 


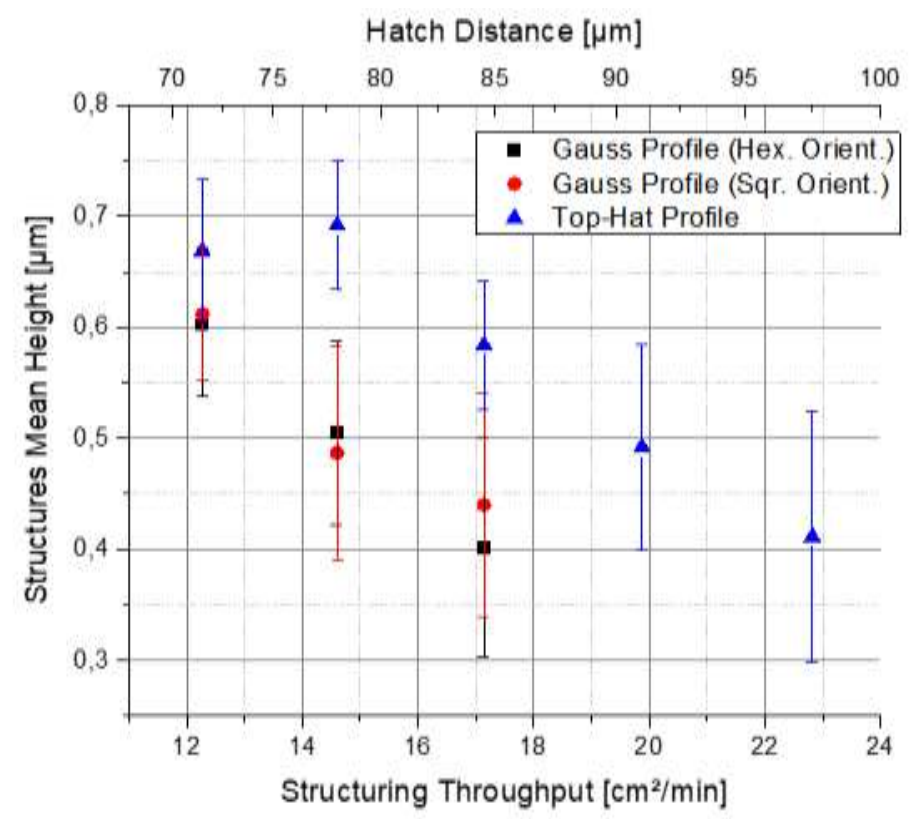

Figure 7. Mean height of produced structures with a filling factor over $80 \%$, structured at $F=2.96 \mathrm{~J} / \mathrm{cm}^{2}$ (laser power: $1.38 \mathrm{~W}$, repetition rate: $4 \mathrm{kHz}$ ).

\section{Conclusions}

In this study, a diffractive beam shaping element, which applies the FBS principle to generate a square-shaped top-hat beam profile, was used in a four-beam DLIP setup. The capabilities of the FBS-DLIP system for laser surface patterning was compared with a standard configuration using a Gaussian beam. Several advantages resulted in this investigation. For example, the structure height could be increased by $\sim 44.8 \%$ when using the top-hat configuration for the same laser fluence. Furthermore, the uniformity could be improved by $\sim 60 \%$ due to the effective redistribution of laser intensity inside the beam. Furthermore, higher fill-factor was obtained for lower hatch distances leading to increased throughput. Finally, it was demonstrated that the productivity could be improved by $\sim 53 \%$ (from 14.5 to $20.0 \mathrm{~cm}^{2} / \mathrm{min}$ ), taking into consideration a periodic pattern with $0.5 \mu \mathrm{m}$ height and over $80 \%$ filling factor.

\section{Acknowledgments}

M.E-K. and A.F.L. acknowledge the German Research Foundation (German: Deutsche Forschungsgemeinschaft DFG) in the framework of the Reinhart-Koselleck project [LA2513 7-1]. M.EK. and T. K. also acknowledge the support funding from the European Union's Horizon 2020 Framework Program for research and innovation under grant agreement no. 768701. T. K. acknowledges the Bundesministerium für Bildung und Forschung (BMBF) for financial support (Verbundförderprojekt "Konzepte zum Aufbau von Elektrodenarchitekturen für Hochenergie- und Hochleistungsbatterien der nächsten Generation," FKZ 03XP0198G).

\section{Author contributions statement}

M.E-K., T.K. and A.F.L. conceived the idea of the work. M.E-K. and B.V. designed the experiments. M.E-K. conducted the experiments.M.E-K. and B.V. analysed the obtained results. M.E-K. prepared the figures. M.E-K., B.V. and A.F.L. wrote the manuscript. All authors reviewed the manuscript and provided critical feedback. A.F.L. supervised the work.

\section{Competing interests}

The authors declare no competing interests.

\section{Data availability}

The datasets generated and analysed during the current study are available from the corresponding author on reasonable request.

\section{References}


1. Milles, S., Soldera, M., Voisiat, B. \& Lasagni, A. F. Fabrication of superhydrophobic and icerepellent surfaces on pure aluminium using single and multiscaled periodic textures. Scientific Reports 9, (2019).

2. Aguilar-Morales, A. I., Alamri, S., Voisiat, B., Kunze, T. \& Lasagni, A. F. The Role of the Surface Nano-Roughness on the Wettability Performance of Microstructured Metallic Surface Using Direct Laser Interference Patterning. Materials 12, 2737 (2019).

3. Milles, S., Soldera, M., Kuntze, T. \& Lasagni, A. F. Characterization of self-cleaning properties on superhydrophobic aluminum surfaces fabricated by direct laser writing and direct laser interference patterning. Applied Surface Science 525, 146518 (2020).

4. Grützmacher, P. G., Profito, F. J. \& Rosenkranz, A. Multi-Scale Surface Texturing in TribologyCurrent Knowledge and Future Perspectives. Lubricants 7, 95 (2019).

5. Helbig, R. et al. The impact of structure dimensions on initial bacterial adhesion. Biomaterials Science 4, 1074-1078 (2016).

6. Lang, V., Voisiat, B. \& Lasagni, A. F. High Throughput Direct Laser Interference Patterning of Aluminum for Fabrication of Super Hydrophobic Surfaces. Materials 12, (2019).

7. Voisiat, B., Ströbel, J., Du, K. \& Lasagni, A. F. How to improve throughput in direct laser interference patterning: top-hat beam profile and burst mode. in Laser-based Micro- and Nanoprocessing XIV 11268, 1-7 (SPIE Digital Library, 2020).

8. Kunze, T. et al. Development of a scanner-based direct laser interference patterning optical head: new surface structuring opportunities. in Laser-based Micro- and Nanoprocessing XI 10092, 1-7 (SPIE Digital Library, 2017).

9. Nakata, Y., Yoshida, M., Osawa, K. \& Miyanaga, N. Fabricating a regular hexagonal lattice structure by interference pattern of six femtosecond laser beams. Appl. Surf. Sci. 417, 69-72 (2017).

10. Indrisiunas, S., Voisiat, B., Žukauskas, A. \& Račiukaitis, G. Direct laser beam interference patterning technique for fast high aspect ratio surface structuring. In Laser Applications in Microelectronic and Optoelectronic Manufacturing (LAMOM) XX 9350, 935003 (International Society for Optics and Photonics, 2015).

11. Lasagni, A. F. et al. Direct laser interference patterning, 20 years of development: from the basics to industrial applications. Laser-based Micro- and Nanoprocessing XI 10092, 1-11 (SPIE Digital Library, 2017).

12. Hunga, Y.-J., Chang, H.-J., Chang, P.-C., Lin, J.-J. \& Kao, T.-C. Employing refractive beam shaping in a Lloyd's interference lithography system for uniform periodic nanostructure formation. Journal of Vacuum Science \& Technology B 35, 1-9 (2017).

13. Indrišiūnas, S., Voisiat, B., Gedvilas, M. \& Račiukaitis, G. New opportunities for custom-shape patterning using polarization control in confocal laser beam interference setup. Journal of Laser Applications 29, 011501 (2017).

14. Lasagni, A. F. et al. High-speed surface structuring using Direct Laser Interference Patterning fundamentals, applications and technology transfer. In Proceedings of LPM2017 - the 18th International Symposium on Laser Precision Microfabrication 1-7 (2017).

15. Mücklich, F. T., Lasagni, A. F. \& Daniel, C. Laser interference metallurgy-periodic surface patterning and formation of intermetallics. Intermetallics 13, 437-442 (2005).

16. Lasagni Andrés Fabián. Advanced design of periodical structures by laser interference metallurgy in the micro/nano scale on macroscopic areas. (Shaker, 2007).

17. Volkov, A. N. \& Zhigilei, L. V. Melt dynamics and melt-through time in continuous wave laser heating of metal films: Contributions of the recoil vapor pressure and Marangoni effects. International Journal of Heat and Mass Transfer 112, 300-317 (2017).

18. Aguilar-Morales, A. I., Alamri, S., Kunze, T. \& Lasagni, A. F. Influence of processing parameters on surface texture homogeneity using Direct Laser Interference Patterning. Optics \& Laser Technology 107, 216-227 (2018). 
19. Bischoff, C. et al. Design and Manufacturing Method of Fundamental Beam Mode Shaper for Adapted Laser Beam Profile in Laser Material Processing. Materials 12, 1-13 (2019).

20. Raciukaitis, G. et al. Laser Processing by Using Diffractive Optical Laser Beam Shaping Technique . Journal of Laser Micro / Nanoengineering 6, 37-43 (2011).

21. Du, K. Thin layer ablation with lasers of different beam profiles - Energy efficiency and over filling factor . in Laser-based Micro- and Nanopackaging and Assembly III 7202, 1-9 (SPIE Digital Library, 2009).

22. $\mathrm{Du}, \mathrm{K}$. "Green processing" of thin film with top-hat lasers and applications in photovoltaic . in Laser-based Micro- and Nanopackaging and Assembly IV 7585, 1-8 (SPIE Digital Library, 2010).

23. Rung, S., Barth, J. and Hellmann, R. Characterization of laser beam shaping optics based on their ablation geometry of thin films, Micromachines 5, 943-953 (2014).

24. Katz , S., Kaplan, N. \& Grossinger, I. Using Diffractive Optical Elements-DOEs for beam shaping - fundamentals and applications. Optik \& Photonik 13, 83-86 (2018).

25. Bischoff, C., Jäger, E. \& Umhofer, U. Beam Shaping Optics for Process Acceleration-Increasing the productivity of laser micromachining. Laser Technik Journal 3, 53-57 (2015).

26. El-Khoury, M., Voisiat, B., Kunze, T. and Lasagni, A. F. Utilizing Fundamental Beam-Mode Shaping Technique for Top-Hat Laser Intensities in Direct Laser Interference Patterning, Journal of Laser Micro/Nanoengineering 13, 268-272 (2018).

27. Roch, T., Benke, D., Lasagni, A.F. Method and Arrangement for Forming a Structuring on Surfaces of Components by Means of a Laser Beam. US 9,764,424 B2, 2017.

28. El-Khoury, M., Voisiat, B., Kunze, T. \& Lasagni, A. F. Prediction of Optimum Process Parameters Fabricated by Direct Laser Interference Patterning Based on Central Composite Design. Materials 13, 1-19 (2020).

29. Liu, J. M. Simple technique for measurements of pulsed Gaussian-beam spot sizes. Optics Letters 7, 196 (1982).

30. Nicol'o, M. et al. Choroidal Vascular Flow Area in Central SerousChorioretinopathy Using Swept-Source Optical CoherenceTomography Angiography. Investigative ophthalmology \& visual science 58, 2002-2010 (2002).

31. Voisiat, B., Zwahr, C. \& Lasagni, A. F. Growth of regular micro-pillar arrays on steel by polarization-controlled laser interference patterning. Applied Surface Science 471, 1065-1071 (2019).

32. Fu, Y., Soldera, M., Wang, W., Voisiat, B. \& Lasagni, A. F. Picosecond Laser Interference Patterning of Periodical Micro-Architectures on Metallic Molds for Hot Embossing. Materials 12, 1-16 (2019).

33. El-Khoury, M., Alamri, S., Voisiat, B., Kunze, T. \& Lasagni, A. F. Fabrication of hierarchical surface textures using multi-pulse direct laser interference patterning with nanosecond pulses. Materials Letters 258, 126743 (2020) 


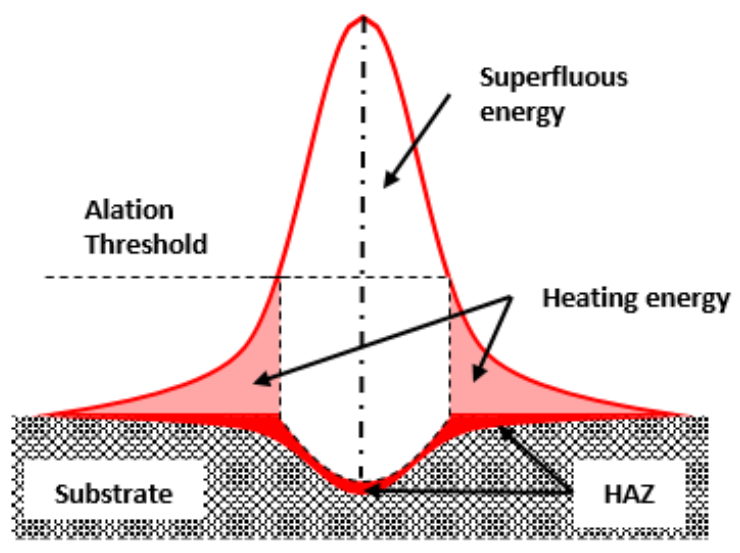

(a) Laser gaussian beam profile on substrate surface

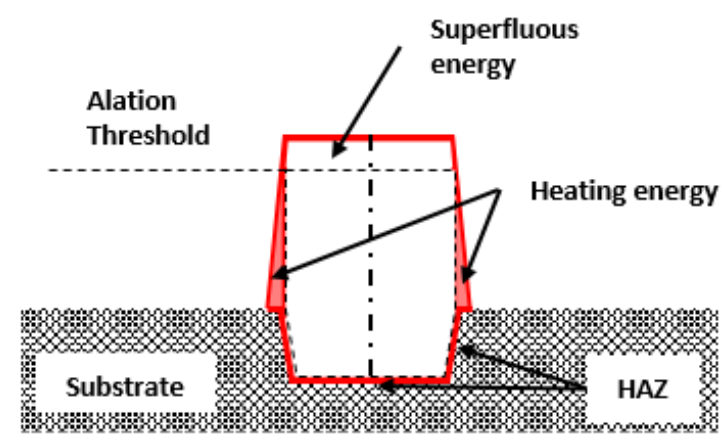

(b) Laser top-hat beam profile on substrate surface
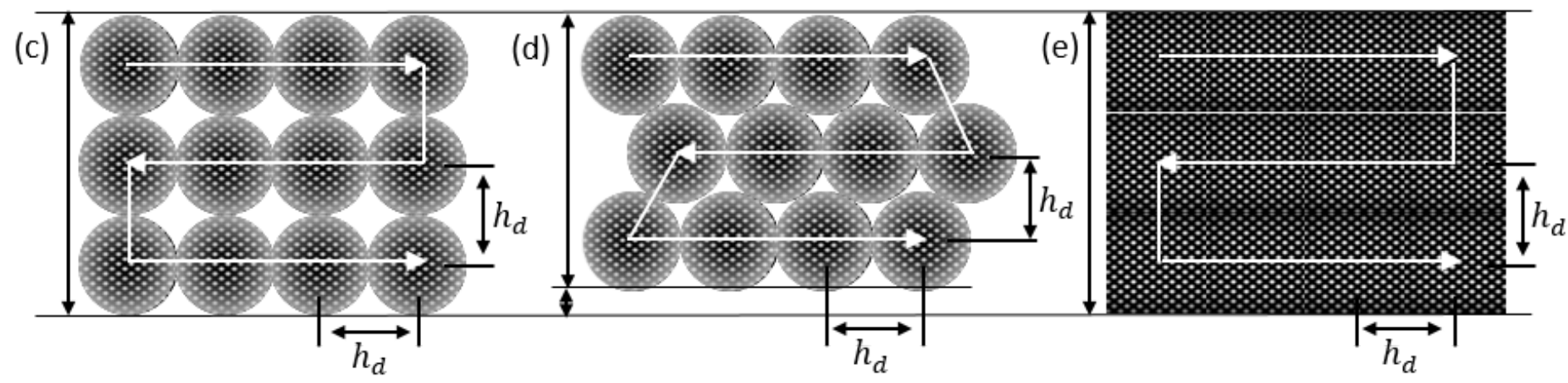

Figure 1

Laser energy contribution to material ablation and formation of Heat affected Zone (HAZ) when (a) Gaussian and (b) top-hat beams are utilized23. Illustration of structured area coverage during DLIP with (c) square and (d) hexagonally distributed Gaussian laser spots as well as (e) top-hat shaped laser beam. hd denotes a hatch distance between two consecutive pulses. 
(a)

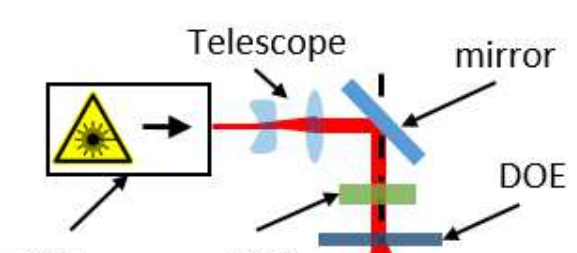

USP Laser

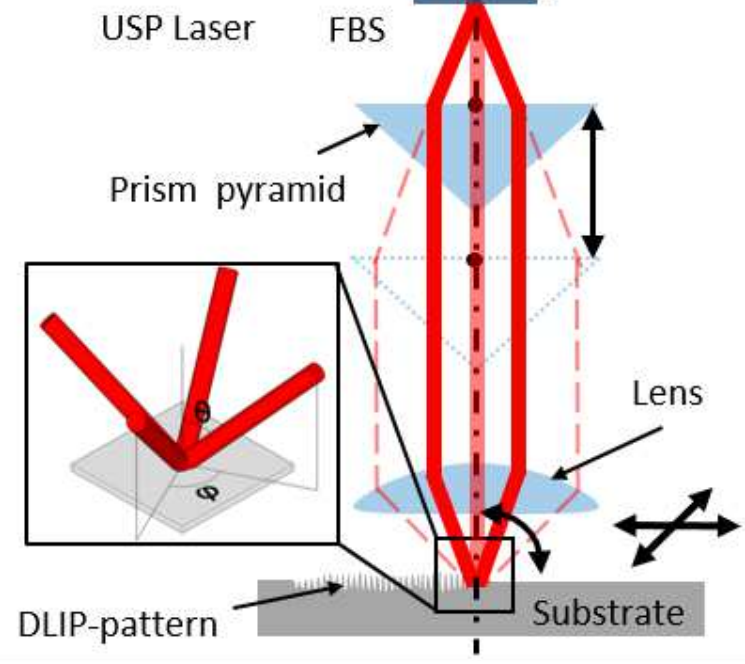

(b)

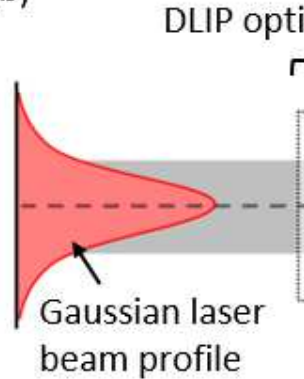

(c)

DLIP optics configuration

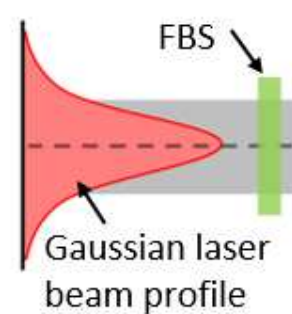

Focused gaussian laser beam with interference modulation

\section{Figure 2}

See the Supplemental Files section for the complete figure caption

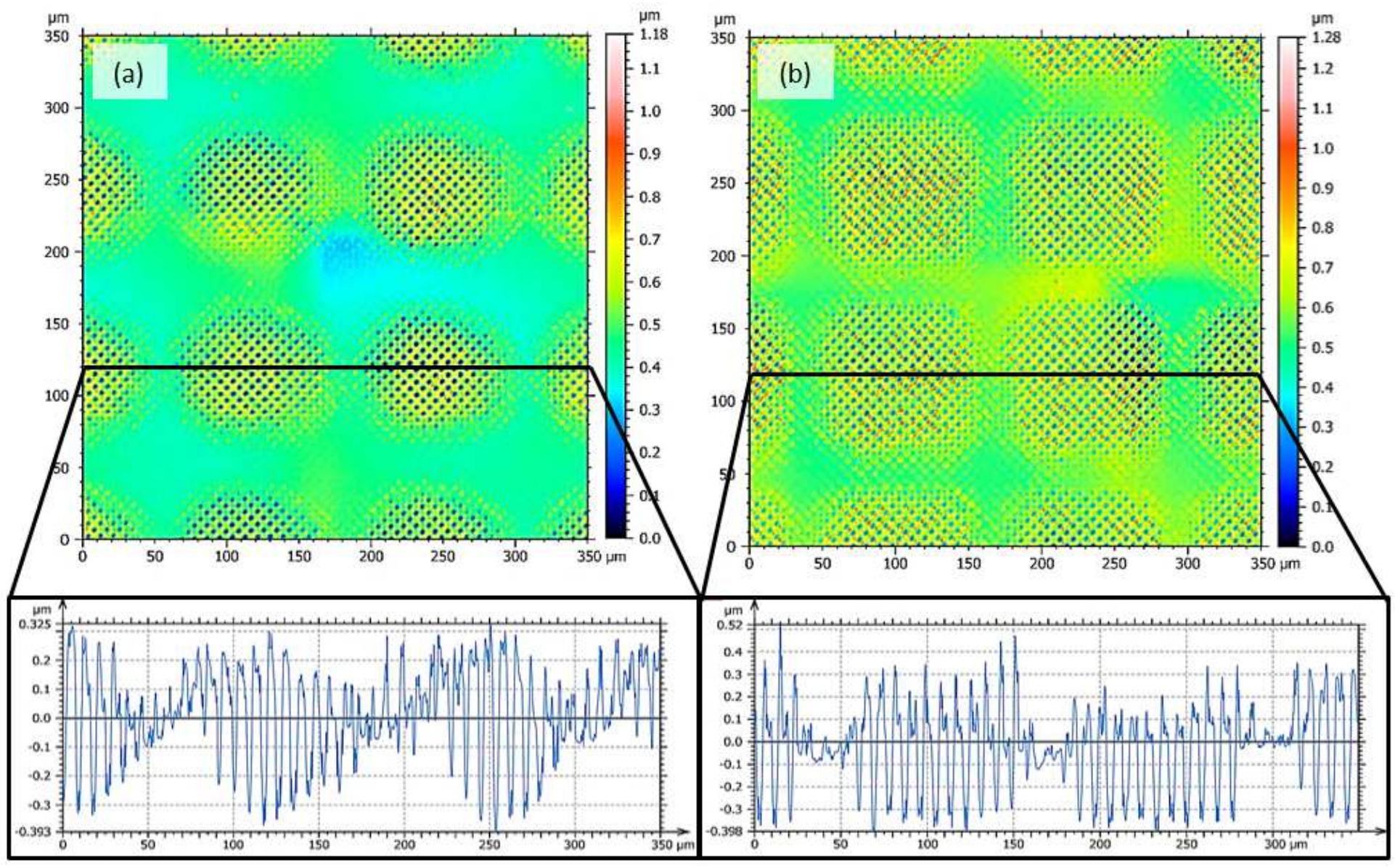

Figure 3 
Confocal microscope images of surface topographies produced in stainless steel and corresponding surface profiles using the (a) Gaussian and (b) top-hat beam profiles. Only one laser pulse was used for each spot. The laser fluence was $2.97 \mathrm{~J} / \mathrm{cm}^{2}$ and the hatch distance was $130 \mu \mathrm{m}$.

(a)

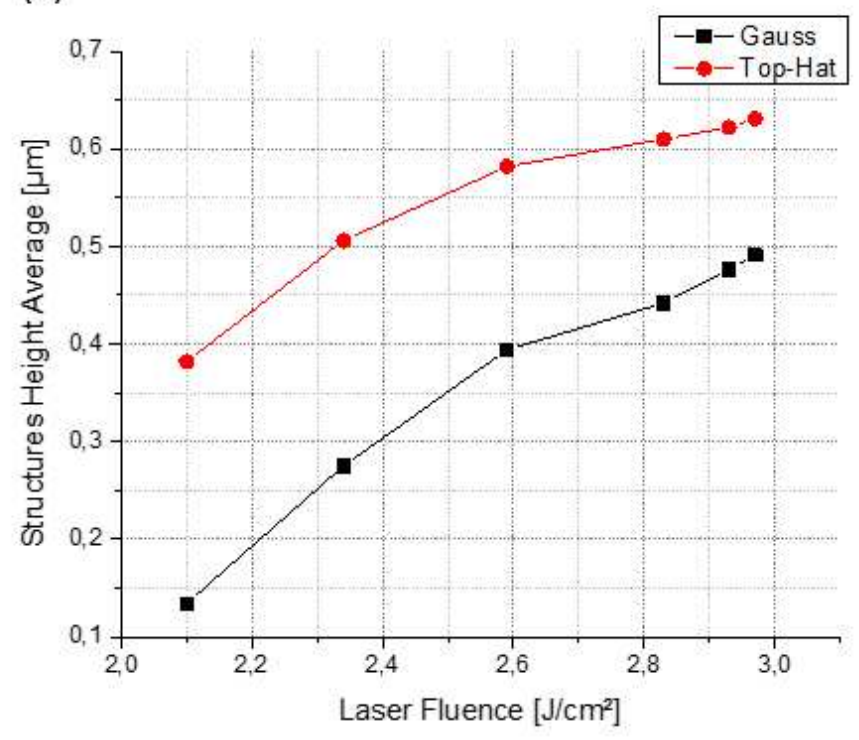

(b)

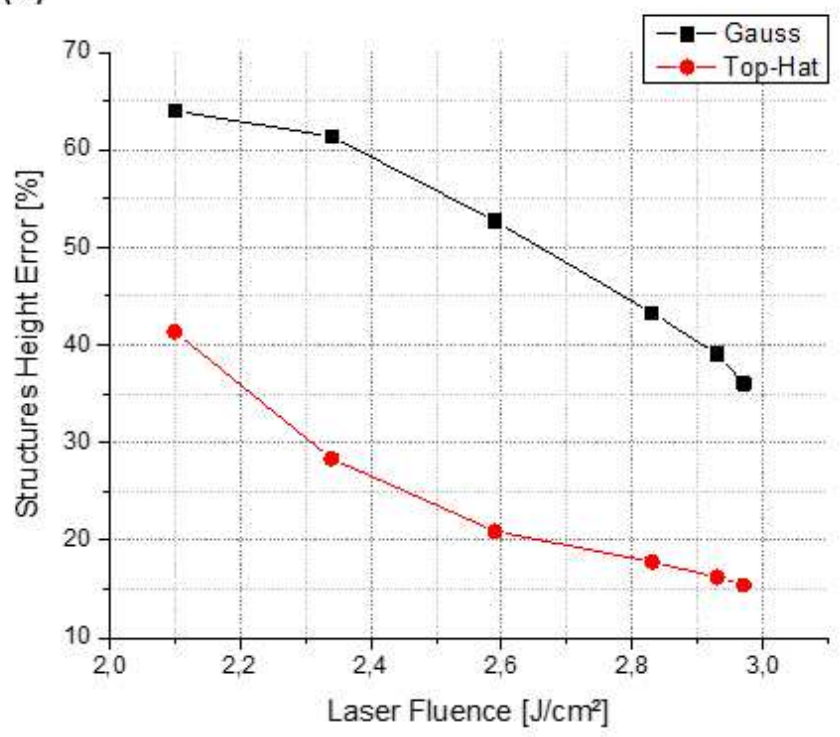

(c)

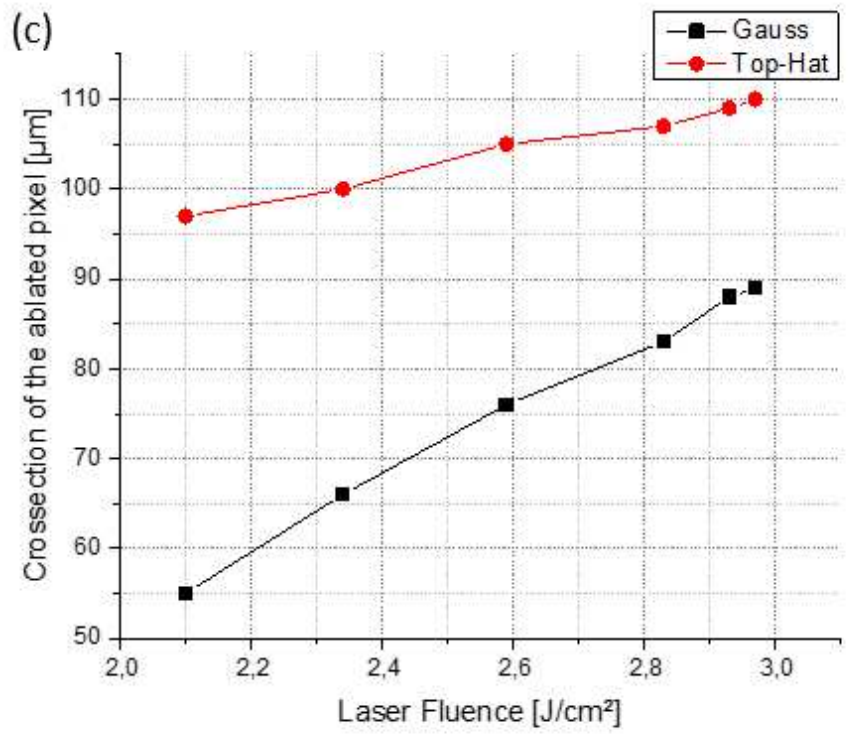

Figure 4

Graphs with topography characteristics of patterns structured with Gaussian beam and Top-hat beam: (a) average structures height, (b) structures height error, (c) size of the ablated spot at different laser fluence values. The lines are used for eye guiding. 
Gaussian, Square Orientation

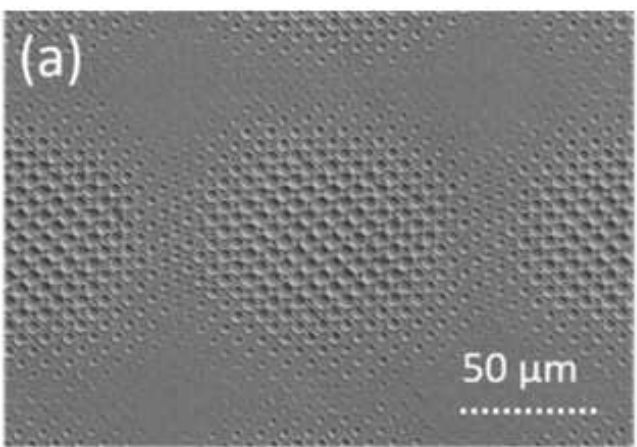

(d)

$50 \mathrm{~km}$
Gauss, Triangular Orientation
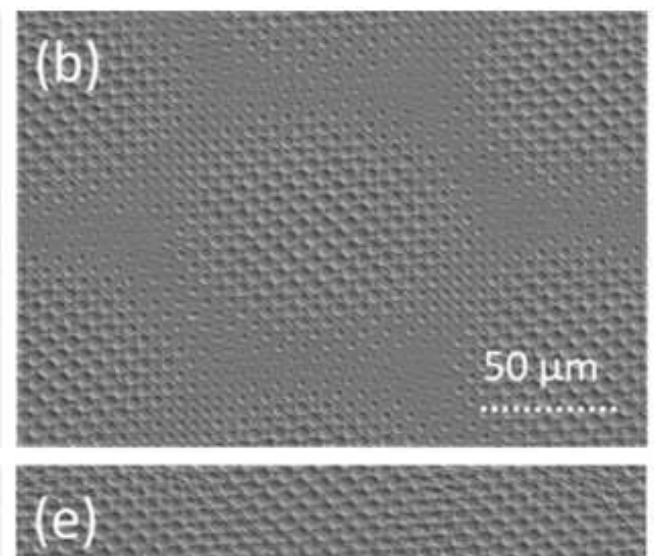

$50 \mu \mathrm{m}$
Top-Hat
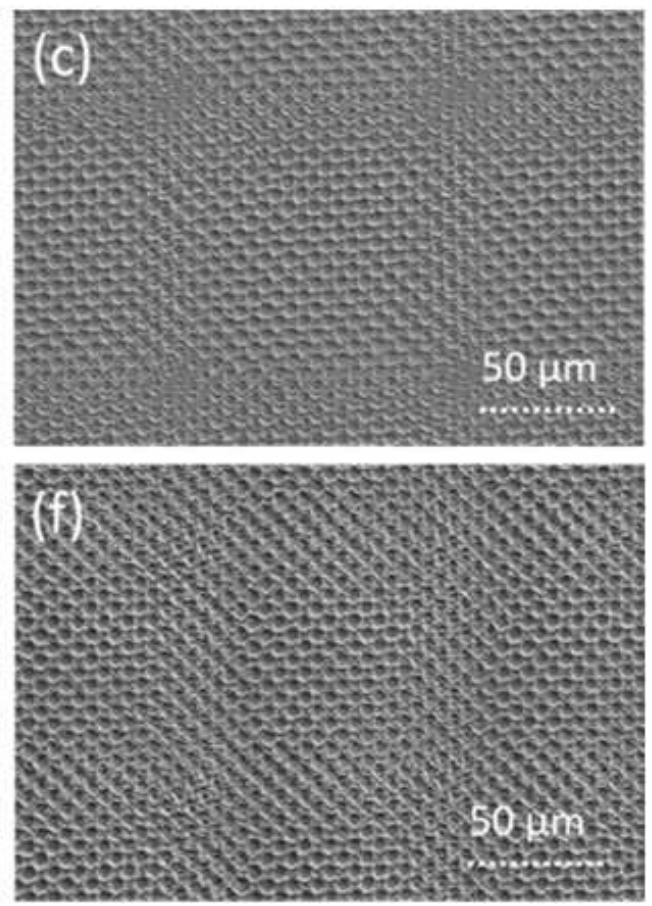

\section{Figure 5}

Scanning electron microscope (SEM) images of textured surfaces using the nanosecond four-beam DLIP configuration, produced on 1.4301 steel. The spatial period $\Lambda$ was $6.50 \mu \mathrm{m}$ and the following process parameters were used: $(\mathrm{a}-\mathrm{c}) \mathrm{F}=2.59 \mathrm{~J} / \mathrm{cm} 2, \mathrm{hd}=91.0 \mu \mathrm{m} ;(\mathrm{d}-\mathrm{f}) \mathrm{F}=2.97 \mathrm{~J} / \mathrm{cm} 2$, hd $=78.0 \mu \mathrm{m}$. The patterns were produced using $(a, d, c, f)$ the square and $(b, e)$ triangular distribution of the laser spots with $(a, b, d, e)$ Gaussian and (c, f) top-hat configurations. 


\section{Gaussian, Square Orientation Gaussian, Triangular Orientation Top-Hat, Square Orientation}
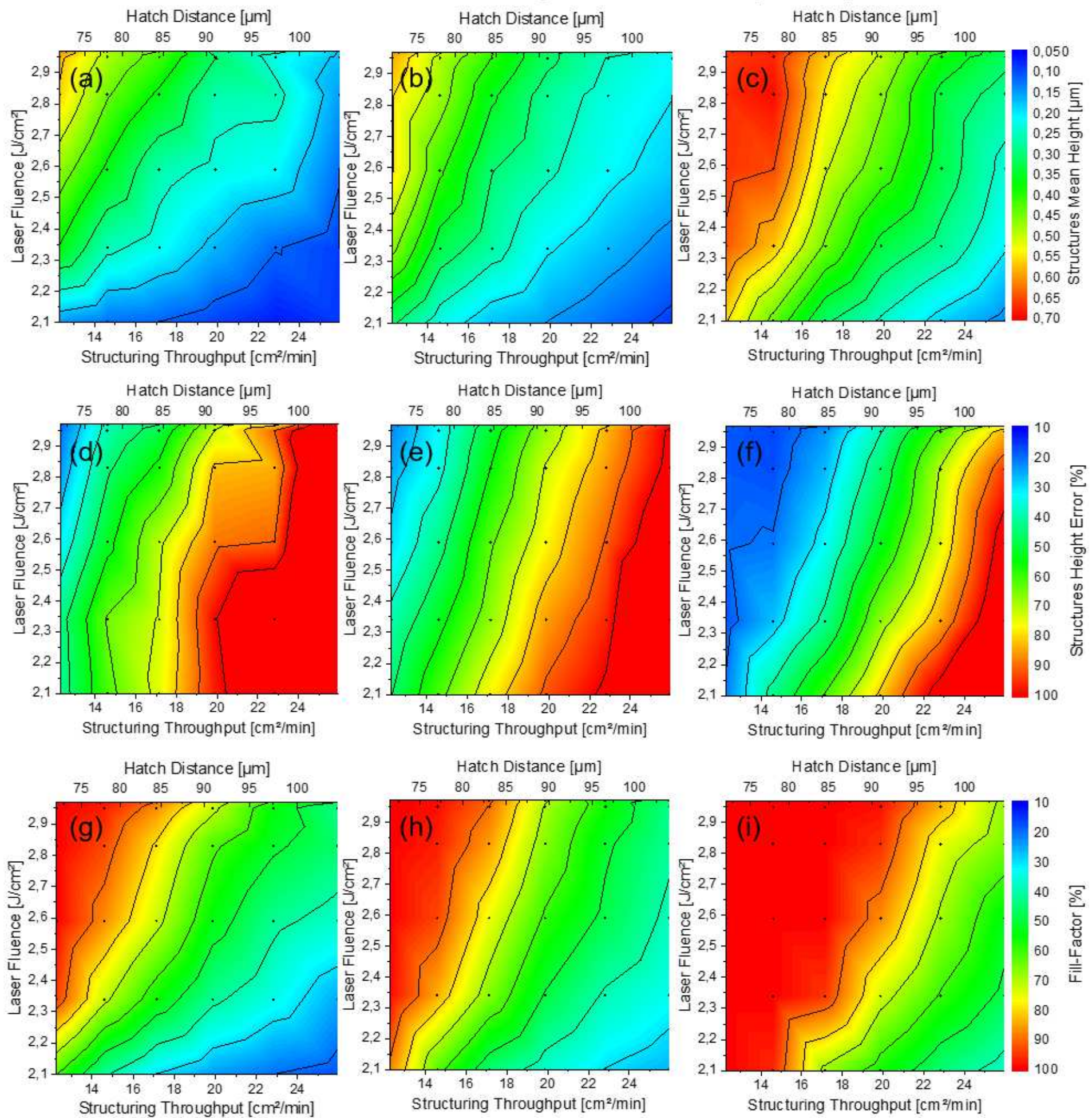

\section{Figure 6}

Contour plots of topography characteristics (structures mean height, structures height error and fill-factor) of the patterned surface with Gaussian and top-hat beam profiles as a function of laser fluence and hatch distance. 


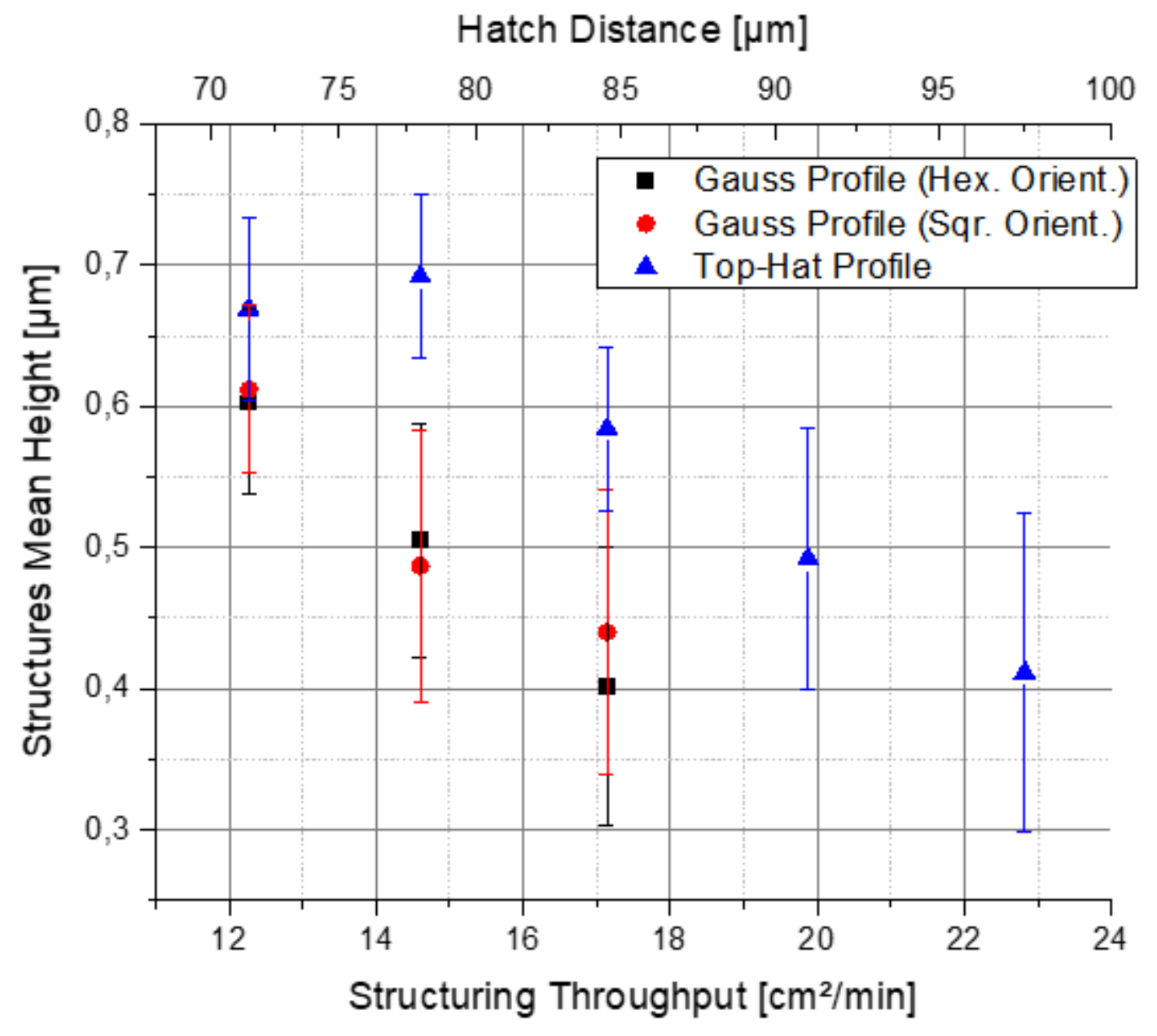

Figure 7

Mean height of produced structures with a filling factor over $80 \%$, structured at $F=2.96 \mathrm{~J} / \mathrm{cm}^{2}$ (laser power: $1.38 \mathrm{~W}$, repetition rate: $4 \mathrm{kHz}$ ).

\section{Supplementary Files}

This is a list of supplementary files associated with this preprint. Click to download.

- Figure02Caption.doc 